\title{
Thyroid Texture Representation via Noise Resistant Image Features
}

\author{
Eystratios G. Keramidas ${ }^{1}$, Dimitris K. Iakovidis ${ }^{1}$, Dimitris Maroulis ${ }^{1}$, \\ and Nikos Dimitropoulos ${ }^{2}$ \\ ${ }^{1}$ Dept. of Informatics and Telecommunications, University of Athens, Greece \\ ${ }^{2}$ Dept. of Medical Imaging, EUROMEDICA Medical Center, Athens, Greece. \\ e.keramidas@di.uoa.gr,dimitris.iakovidis@ieee.org,dmarou@di.uoa.gr
}

\begin{abstract}
The robustness of textural features on speckle noise is of vital importance for ultrasound imaging. A set of novel fuzzy features for thyroid ultrasound texture representation, demonstrating noise-resistant properties, is presented, analyzed and evaluated in this study. The textural feature extraction scheme is based on the fuzzyfication of the local binary pattern approach. The proposed features are evaluated on an annotated dataset of B-mode thyroid ultrasound images acquired from 75 patients. The experimental results illustrate that these features provide accurate representation of the thyroid texture. They can be effectively utilized for thyroid nodule detection outperforming other thyroid texture representation approaches that have been recently proposed in the literature.
\end{abstract}

\section{Introduction}

Ultrasonography is one of several diagnostic methods currently available for the evaluation of the thyroid gland. It combines a unique set of virtues including real time imaging, ability to measure dimensions and volume, low cost, mobility, and no side effects. As a result ultrasonography has become the most widely employed imaging method for the diagnosis and follow-up of thyroid disorders such as nodules, tumors and cysts [1-4].

However, an inherent characteristic of ultrasound imaging is the presence of multiplicative speckle noise

This work was supported by the Greek General Secretariat of Research and Technology (25\%), the European Social Fund (75\%), and private sector, under the framework of Measure 8.3 of E.P. Antagonistikotita - 3rd European Support Framework - PENED 2003 (grant no. 03-ED-662). This work was also partially funded by Special Account for Research Grants of the National and Kapodistrian University of Athens.
[5]. Speckle noise generally tends to reduce the image resolution and contrast, inducing a degree of uncertainty. Therefore medical diagnosis based on the assessment of ultrasound images can be greatly affected by this type of image degradation.

Several endeavors were undertaken to improve interpretation of thyroid ultrasound images through quantitative criteria. Early studies on texture analysis of the thyroid gland [6-8] were limited to features extracted from grey-level histograms. Latter works [9][10] utilized features extracted from co-occurrence matrix [11] and intensity based information, for the analysis of thyroid tissue in ultrasound images. Other studies on texture classification [12] and segmentation [13] of nodular thyroid tissue, have been based on texture representation via Local Binary Patterns (LBP) [14] approach. Thyroid tissue directionality has also been explored in [15] where Radon domain features have been considered to encode the directionality patterns in thyroid ultrasound images. However, most texture representation approaches mentioned above, tend to exhibit susceptibleness to noise and artifacts appearing in ultrasound imaging.

The presence of speckle noise and artifacts in thyroid ultrasound images necessitates the use of a noise resistant texture descriptor. The purpose of this study is to present and evaluate a set of novel textural features capable of coping with the uncertainty inherent to ultrasound thyroid images. Based on the fact that fuzzy theory provides a mathematical framework to coping with uncertainty we derive noiseresistant Local Binary Pattern texture features, called Fuzzy Local Binary Pattern (FLBP) features.

This paper is organized as follows. In Section 2, the proposed FLBP feature extraction method is presented. In Section 3, real thyroid ultrasound images are utilized for a comparative experimental evaluation of the proposed FLBP method and other feature extraction 


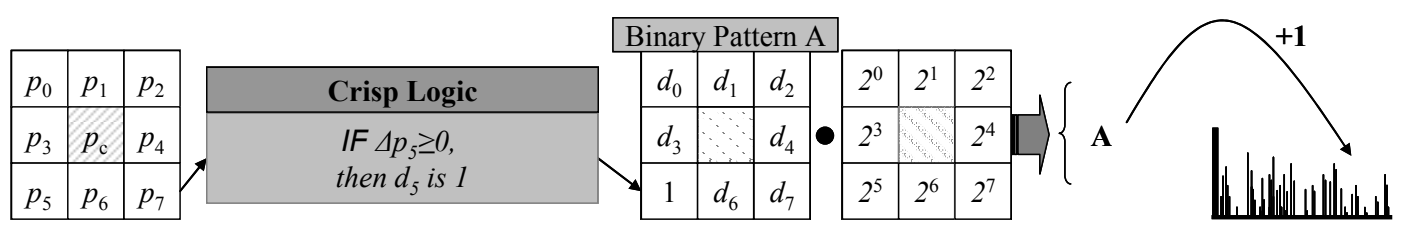

(a)

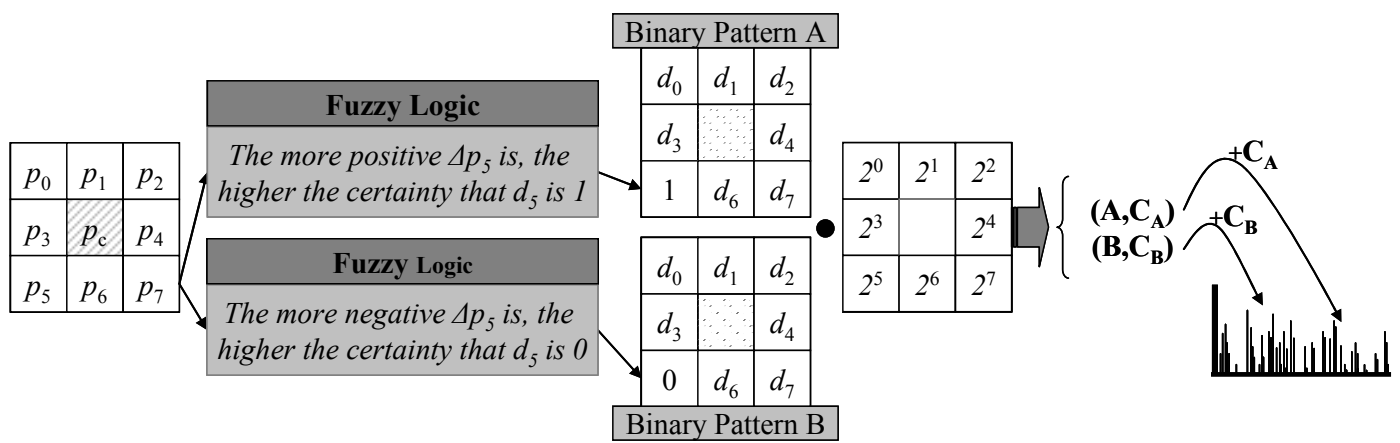

(b)

Figure 1. (a) LBP computation scheme. (b) Example of the FLBP computation scheme, where for every $i !=5$, $-F>\Delta p_{i}$ or $\Delta p_{i}>F$, and for $i=5,-F<\Delta p_{i}<F$.

methods. Finally Section 4, summarizes experimental results and presents conclusions.

\section{Proposed Texture Representation}

The local binary pattern (LBP) [14] is a popular approach for texture representation, showing excellent results in many empirical studies. Derived from a general definition of texture, based on the concept of texture primitives, often called texels or textons, the LBP approach provides a theoretically and computationally simple and efficient methodology for texture analysis.

To represent the formations of a textured image, the LBP approach, models $3 \times 3$ textons (Fig. 1a). A $3 \times 3$ texton consists of a set of nine elements, $P=\left\{p_{\text {center },} p_{0}\right.$, $\left.p_{1}, \ldots, p_{7}\right\}$, where $p_{c}$ represents the intensity value of the central pixel and $p_{\mathrm{i}}(0 \leq i \leq 7)$ represent the intensity values of the peripheral pixels (Fig. 1a). Each texton then, can be characterized by a set of binary values $d_{i}(0 \leq i \leq 7)$ (Fig. 1b), where

$$
d_{i}= \begin{cases}1 & \text { if } \Delta p_{i} \geq 0 \\ 0 & \text { if } \Delta p_{i}<0\end{cases}
$$

where $\Delta p_{\mathrm{i}}=p_{\mathrm{i}}-p_{\text {center }}$.
For each $3 \times 3$ texton a unique LBP code can be derived by these binary values, as follows:

$$
L B P=\sum_{i=0}^{7} d_{i} \cdot 2^{i}
$$

Every pixel in an image generates such an LBP code (Fig. 1a). A single LBP code represents the local microtexture information around a pixel by a single integer code $L B P \in[0,255]$. Then a histogram is created to collect up the occurrences of different LBP codes from all pixels in the image. This histogram forms the LBP feature vector, which characterizes the image texture.

The LBP approach is based on Boolean logic through hard thresholding of peripheral pixels. This characteristic makes LBP texture representation sensitive small variations of pixel intensities similar to effects caused by noise. To enhance the LBP approach so as to cope with such variations and thus uncertainty introduced by the speckle noise in ultrasound images, we have considered fuzzy logic. Fuzzy logic has certain major advantages over traditional Boolean logic when it comes to real world applications such as texture representation of real images. Introduced by L.A. Zadeh in (1965) [16], fuzzy theory excels in 


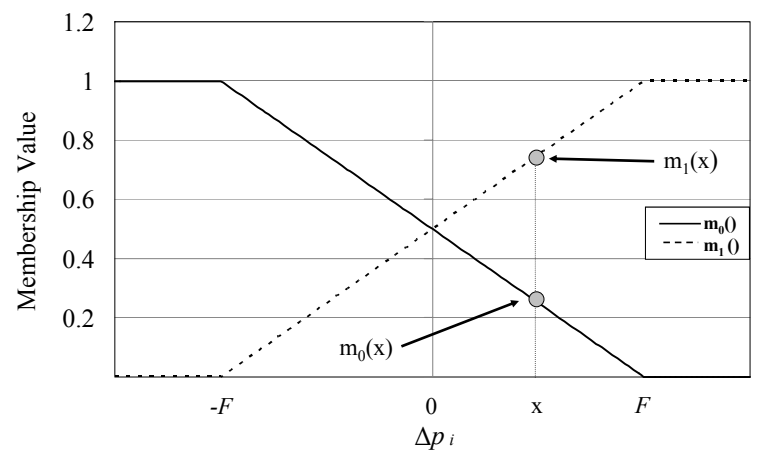

Figure 2. Membership functions $m_{0}()$ and $m_{1}()$ as a function of $\Delta p_{i}$.

producing exact results from imprecise data. The main difference between the fuzzy and the classic logic is that statements are no longer 0 or 1 , but assume any real value between 0 and 1, that allows more humanlike interpretation and reasoning.

The incorporation of fuzzy logic in the LBP approach includes the transformation of the input variables to respective fuzzy variables, according to a set of fuzzy rules. We define two linguistic rules to describe the relation between the intensity values of the peripheral pixels $p_{i}$ and the center pixel $p_{c}$ of a $3 \times 3$ neighborhood in a more human-like fashion, as follows:

\section{Rule 0:}

The more negative $\Delta p_{\mathrm{i}}$ is, the greater the certainty that $d_{i}$ is 0 .

Rule 1:

The more positive $\Delta p_{\mathrm{i}}$ is, the greater the certainty that $d_{i}$ is 1 .

Based on these two rules two membership functions $m_{0}()$ and $m_{1}()$ can be defined. Let function $m_{0}()$ define the degree to which $\Delta p_{\mathrm{i}}$ is negative, i.e. the degree to which $d_{i}$ is 0 . As a membership function $m_{0}()$ we consider a decreasing function (Fig. 2) defined as follows:

$$
m_{0}(i)= \begin{cases}0 & \text { if } \Delta p_{i} \geq F \\ \frac{F-\Delta p_{i}}{2 \cdot F} & \text { if }-F<\Delta p_{i}<F \\ 1 & \text { if } \Delta p_{i} \leq-F\end{cases}
$$

Similarly $m_{1}()$ is an increasing function that defines the degree to which $\Delta p_{\mathrm{i}}$ is positive, i.e. the degree to which $d_{i}$ is 1 . As membership function $m_{1}()$ we consider the following (Fig. 2):

$$
m_{1}(i)= \begin{cases}1 & \text { if } \Delta p_{i} \geq F \\ \frac{F+\Delta p_{i}}{2 \cdot F} & \text { if }-F<\Delta p_{i}<F \\ 0 & \text { if } \Delta p_{i} \leq-F\end{cases}
$$

For both $m_{0}()$ and $m_{1}(), \quad F \in(0,255]$ represents a parameter that controls the degree of fuzziness.

Contrary to what stated above for the original LBP operator, where a single LBP code characterize a $3 \times 3$ texton, in the proposed FLBP approach, a texton can be characterized by more than one LBP codes. Figure 1(b) presents an example of the FLBP approach, where two LBP codes $(\mathrm{A}, \mathrm{B})$ characterize a $3 \times 3$ texton. The degree to which each LBP code characterize a texton $\left(\mathrm{C}_{\mathrm{A}}, \mathrm{C}_{\mathrm{B}}\right)$, depends on the values of the membership functions $m_{0}()$ and $m_{1}()$ that corresponds to each peripheral pixel. For a $3 \times 3$ texton, the contribution $C_{L B P}$ of each LBP code in the FLBP histogram is defined as:

$$
C_{L B P}=\prod_{i=0}^{8} m_{d_{i}}(i)
$$

where $d_{i} \in\{0,1\}$ and LBP code can be obtained from Eq. 2. Each $3 \times 3$ texton contributes to more than one bins of the FLBP histogram and always the total contribution of a single neighbourhood is

$$
\sum_{L B P=0}^{255} C_{L B P}=1
$$

\section{Results}

The thyroid ultrasound images used in the experiments conducted during this study were provided by the Euromedica Medical Center, Athens, Greece. Ultrasound examinations were performed on 75 patients, using a digital ultrasound system Philips HDI 5000 with a 5-12 MHz linear transducer. All the ultrasound images were stored in digital imaging and communications in medicine (DICOM) format. From each image an expert radiologist selected a balanced set of 32×32-pixel square blocks from normal and nodular tissue (Fig. 3). A total of 250 tissue samples constitute a balanced dataset for training and testing. 


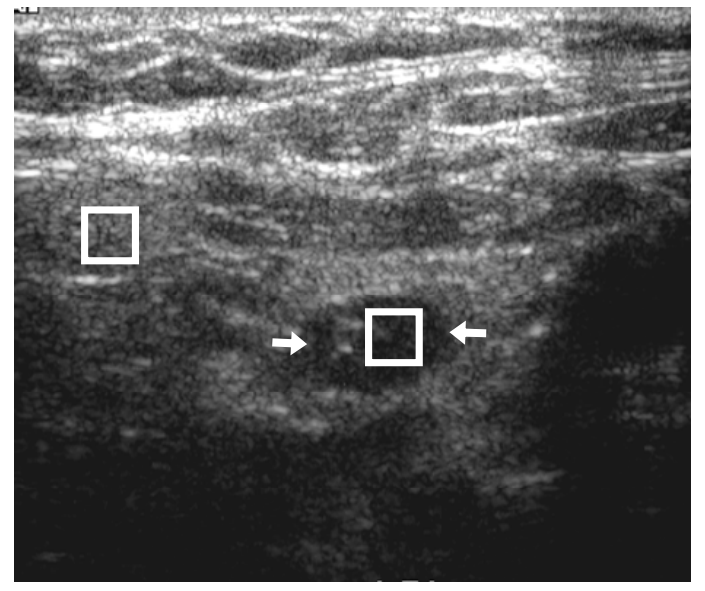

(a)

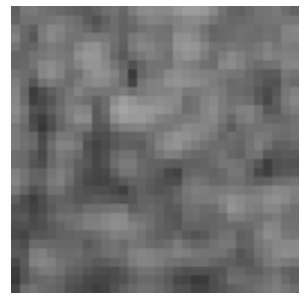

(c)

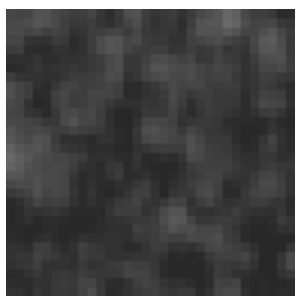

(d)

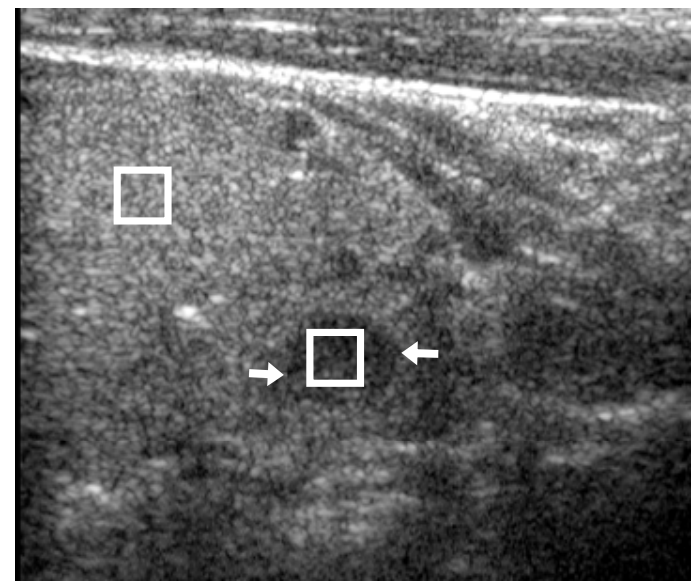

(b)

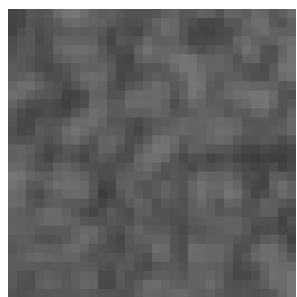

(e)

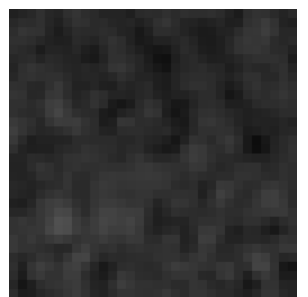

(f)

Figure 3. (a)(b) Representative thyroid ultrasound images with visible nodules marked with arrows. (c)(e) Square blocks sampled from normal parenchyma of images (a) and (b) respectively. (d)(f) Square block sampled from nodular tissue of images (a) and (b) respectively.

For the supervised classification phase of the experimental procedure the Support Vector Machine(SVM) [17] algorithm has been adopted. Due to its strong theoretical foundation based on the principle of structural risk minimization, SVM provides good generalization performance and perform rather well in higher dimensional spaces. To optimize the performance of the SVM classification algorithm a polynomial kernel function has been utilized. The

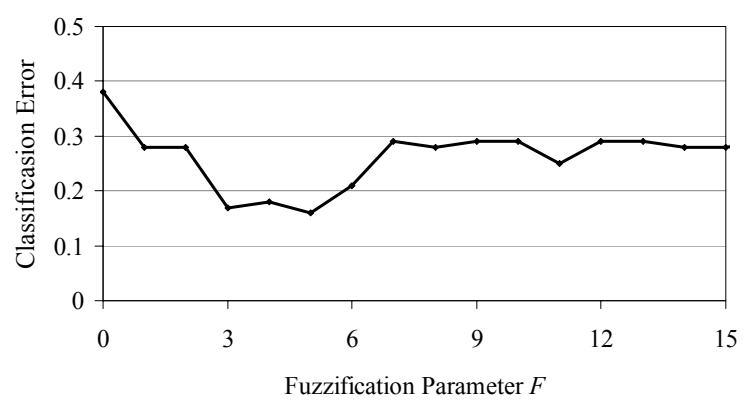

Figure 4. Mean classification error for different values of fuzzification parameter $F \in[1,15]$. For $F=0$ results correspond to crisp LBP approach. classification accuracy for all experiments was estimated by 10 -fold cross validation [18].

Initially a set of experiments have been conducted for the evaluation of the performance improvement

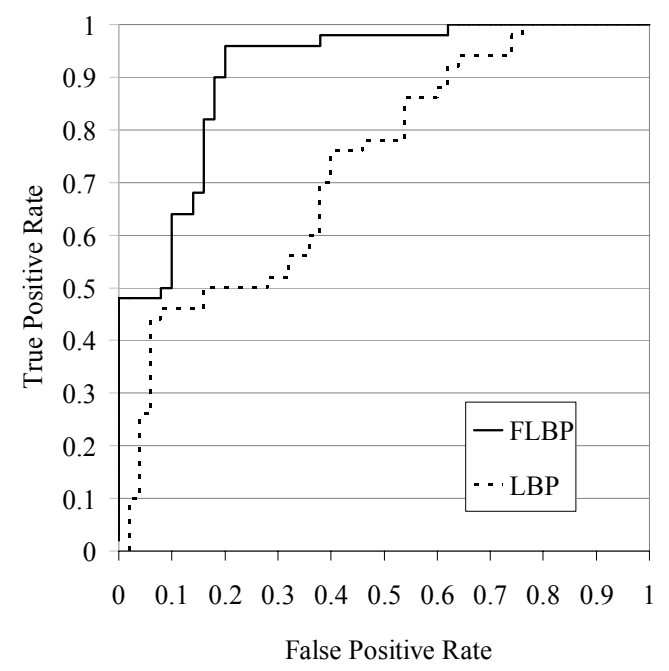

Figure 5. ROC curves obtained with LBP and with FLBP feature vectors for $F=5$. 
achieved by the proposed FLBP approach compared with the original LBP approach. For fuzzification parameter $F$ ranging within [1, 15], fifteen FLBP feature sets were extracted and classified using the SVM algorithm. Additionally, the crisp LBP features $(F=0)$ have been extracted and classified in a similar manner. The classification errors for each of these experiments are illustrated in Figure 4. From this figure, it can be deduced that the FLBP outperforms the LBP approach for every $F$ value tested. The mean classification error obtained by using the LBP features was $38 \%$ whereas for the FLBP a minimum mean classification error of $16 \%$ was obtained for $F=5$. The performance improvement achieved by using the FLBP instead of the LBP features can also be observed in the ROC curves plotted in Fig. 6. The corresponding sensitivity and specificity estimates for the case of the LBP features were $62 \%$ and $62 \%$ respectively. For the case of the FLBP features they reached $76 \%$ and $92 \%$ respectively.

The application of three feature extraction methodologies proposed in literature for thyroid ultrasound image representation was also investigated. Feature vectors extracted by means of co-occurrence matrices [10] produced a minimum classification error of $33 \%$, with $58 \%$ specificity at $96 \%$ sensitivity. The Radon domain features proposed in [15] produced a minimum classification error of $23 \%$, and a specificity of $72 \%$ at $62 \%$ sensitivity. These experimental results advocate the effectiveness of the FLBP features for thyroid texture representation as compared with the other relevant approaches.

\section{Conclusions}

A novel thyroid texture representation approach based on fuzzy logic and Local Binary Patterns has been presented and evaluated in this study. It is called FLBP and it offers considerably improved texture representation, resistant to the speckle noise that is inherent in thyroid ultrasound images. The experimental evaluation of the FLBP approach on real ultrasound thyroid images in comparison with the crisp LBP, the co-occurrence matrix, and with the Radon transformation approaches has been presented. The results demonstrated a higher classification performance of the FLBP as compared to these approaches. It can be concluded that the proposed approach can be valuable for thyroid ultrasound image analysis and diagnostic systems.

\section{Acknowledgments}

We would like to EUROMEDICA S.A., Greece for the provision of the medical images. We would also like to thank Dr. G. Legakis for his continuous support and advice.

\section{References}

[1] J.T. Bushberg, The Essential Physics of Medical Imaging. Lippincott Williams \& Wilkins, 2002, ISBN 0683301187.

[2] G. Mailloux, M. Bertrand, R. Stampfler, and S. Ethier, "Local histogram information content of ultrasound B-mode echographic texture", Ultrasound in Medicine and Biology, Vol. 11, pp. 743-750, 1985.

[3] G. Mailloux, M. Bertrand, R. Stampfler. and S. Ethier, "Computer Analysis of Echographic Textures in Hashimoto Disease of the Thyroid", Journal of Clinical Ultrasound, Vol. 14, pp. 521-527, 1986.

[4] T. Chikui, K. Okamura, K. Tokumori, S. Nakamura, M. Shimizu, M. Koga, and K. Yoshiura, "Quantitative analyses of sonographic images of the parotid gland in patients with Sjögren's syndrome", Ultrasound in Medicine and Biology, Vol. 32, pp. 617-622, 2006.

[5] S. Jasjit, D. Wilson, and S. Laxminarayan, (Eds.) Handbook of Biomedical Image Analysis, 2005, ISBN: 9780-306-48550-3.

[6] G.E. Mailloux, M. Bertrand, R. Stampfler, and S. Ethier, "Texture analysis of ultrasound B-mode images by segmentation," Ultrason. Imag., Vol. 6, No. 3, pp. 262-277, 1984.

[7] H. Morifuji, "Analysis of Ultrasound B-mode Histogram in Thyroid Tumors", Nippon Geka Gakkai Zasshi, Vol. 90, No. 2, pp. 210-221, 1989.

[8] T. Hirning, I. Zuna and D. Schlaps, "Quantification and classification of echographic findings the thyroid gland by computerized b-mode texture analysis", Eur J Radiol, Vol. 9, No. 4, pp. 244-247, 1989.

[9] S. Tsantis, D. Cavouras, I. Kalatzis, N. Piliouras, N. Dimitropoulos, and G. Nikiforidis, "Development Of A Support Vector Machine-Based Image Analysis System For Assessing The Thyroid Nodule Malignancy Risk", Ultrasound Med Biol, Vol. 31, pp. 1451-9, 2005.

[10] C. Skouroliakou, M. Lyra, A. Antoniou, and L. Vlahos, "Quantitative image analysis in sonograms of the thyroid. gland", Nuclear Instruments and Methods in Physics, Vol. 569, pp. 606-609, 2006. 
[11] R.M. Haralick, I. Dinstein, K. Shanmugamm, "Textural Features for Image Classification”, IEEE Trans. On Systems, Man and Cybernetics, Vol. 3, No. 6, pp. 610-621, 1973.

[12] E.G. Keramidas, D. Iakovidis, D. Maroulis, and S.A. Karkanis, "Efficient and Effective Ultrasound Image Analysis Scheme for Thyroid Nodule Detection", Lecture Notes in Computer Science, Vol. 4633, pp. 1052-1060, 2007. [13] M.A. Savelonas, D.K. Iakovidis, D.E. Maroulis, andS.A. Karkanis, "An Active Contour Model Guided by LBP Distributions", Lecture Notes in Computer Science, Vol. 4179, pp. 197-207, 2006.

[14] T. Ojala, M. Pietikäinen, and D. Harwood, "A comparative study of texture measures with classification based on featured distribution", Pattern Recognition, Vol. 29, pp. 51-59, 1996.
[15] M.A. Savelonas, D.K. Iakovidis, N. Dimitropoulos, and D. Maroulis, "Computational Characterization of ThyroidTissue in the Radon Domain", Proc. IEEE Internationa Symposium on Computer-Based Medical Systems, Slovenia, pp. 189-192, 2007.

[16] L.A. Zadeh, "Fuzzy sets", Information and Control, Vol. 8, pp. 338-353, 1965.

[17] V. Vapnik, Statistical Learning Theory, Wiley, New York, 1998, ISBN: 978-0471030034.

[18] S. Theodoridis, and K. Koutroumbas, Pattern Recognition, Academic Press, (3nd edition), 2006, ISBN: 012-369531-7. 